\title{
DIFFRACTIVE DIJETS AND COMBINED FITS FROM H1
}

\author{
M. U. MOZER \\ Physikalisches Institut, Universität Heidelberg, \\ Philosophenweg 12, \\ 69120 Heidelberg, Germany \\ E-mail: mozer@physi.uni-heidelberg.de
}

\begin{abstract}
I present measurements of dijet cross-sections in diffractive DIS taken with the H1 detector at the HERA accelerator. Diffractive events were identified by a rapidity gap selection. The resulting differential cross sections are compared to QCD calculations in NLO, based on parton densities extracted from inclusive diffraction. Additionally a fit of diffractive parton densities to the combined data sets of the inclusive $F_{2}^{D}$ measurement and the dijet data was performed. This leads to reduced uncertainties for the gluon density.
\end{abstract}

\section{Introduction}

Theoretically it is expected that the cross sections of diffractive deepinelastic scattering (DIS) factorizes into universal diffractive parton distributions and process dependent hard scattering coefficients [1]. Diffractive parton densities have been determined from DGLAP QCD fits to inclusive diffractive HERA data $[2,3]$ and have been found to be dominated by the gluon distribution. Diffractive dijet production is directly sensitive to the gluon component of the diffractive exchange and has been shown for DIS [4] - to be in decent agreement with the QCD fits to the inclusive diffractive data. In this paper, a new measurement of diffractive dijet cross sections in deep inelastic scattering is presented, based on data collected with the H1 detector at HERA. A combined NLO QCD fit is performed to the differential dijet cross sections and the inclusive diffractive structure function $F_{2}^{D}$ in order to determine the diffractive quark and gluon distributions with higher accuracy.

\section{Experimental Procedure}

Differential twojet cross sections in diffractive deep inelastic scattering processes at low $Q^{2}$ have been measured with the H1 detector using an inte- 
grated luminosity of $51.5 \mathrm{pb}^{-1}$. Twojet events are identified using the inclusive $k_{T}$ cluster algorithm in the $\gamma^{\star}-p$ rest frame. The cross sections are given at the level of stable hadrons and correspond to the kinematic range: $4 \mathrm{GeV}^{2}<Q^{2}<80 \mathrm{GeV}^{2}, 0.1<y<0.7, x_{\mathbb{P}}<0.03, M_{Y}<1.6 \mathrm{GeV}$, $|t|<1 \mathrm{GeV}^{2}, p_{\perp, \text { jet } 1}^{\star}>5.5 \mathrm{GeV}, p_{\perp, \text { jet } 2}^{\star}>4 \mathrm{GeV}$ and $-3<\eta_{\text {jet }}^{\star}<0$. Diffractive events are selected by requiring a rapidity gap between the central mass system $X$ and the proton direction.

\section{Results}
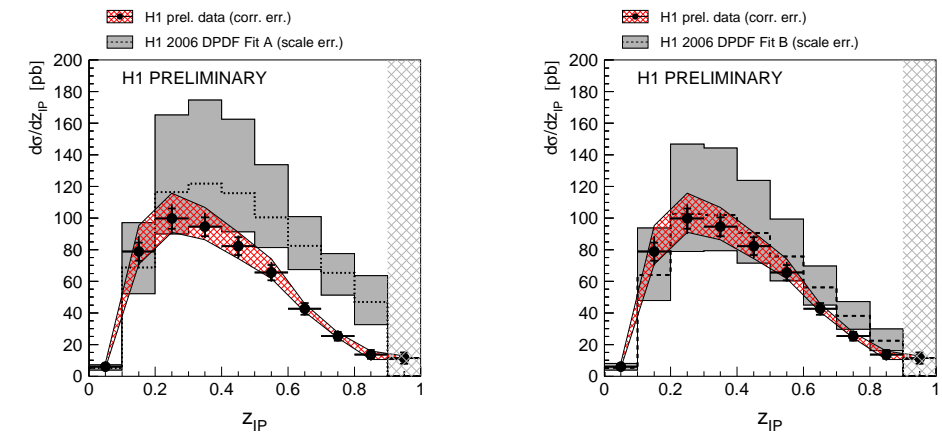

Figure 1. Cross section of diffractive dijets differential in $z_{\mathbb{P}}$ compared to NLO predictions based on the parton-densities from the H1 2006 DPDF fit [3]. The data are shown as black points with the inner and outer error-bar denoting the statistical and uncorrelated systematic uncertainties respectively. The red hatched band indicates the correlated systematic uncertainty. The black line shows the NLO QCD prediction based on the H1 2006 DPDF fit A (left) and H1 2006 DPDF fit B (right) and is surrounded by a grey band indicating the scale uncertainty.

Figure 1 shows the differential dijet cross section compared to NLO predictions based on the parton densities from the fit to the inclusive diffractive data [3]. While the prediction based on the H1 2006 DPDF fit A clearly overestimates the cross section, there is reasonable agreement between the data and the H1 2006 DPDF fit B. Nevertheless, significant discrepancies also exist for the H1 2006 DPDF fit B at high $z_{\mathbb{P}}$.

\section{Combined Fit}

The differential dijet cross section in $z_{\mathbb{P}}$ is used in the fit in 4 bins of the scale variable $p_{\perp}^{\star 2}+Q^{2}$ to constrain the gluon density, where $p_{\perp}^{\star}$ is the 
transverse momentum of the hardest jet. These measured cross sections are shown in Figure 2 (left). Additionally the inclusive data sample of a previous $\mathrm{H} 1$ analysis [3] is used to constrain the quark density and the gluon density a low momentum fraction. A part of the $F_{2}^{D}$ measurements is shown in Figure 2 (right) together with the final NLO prediction.

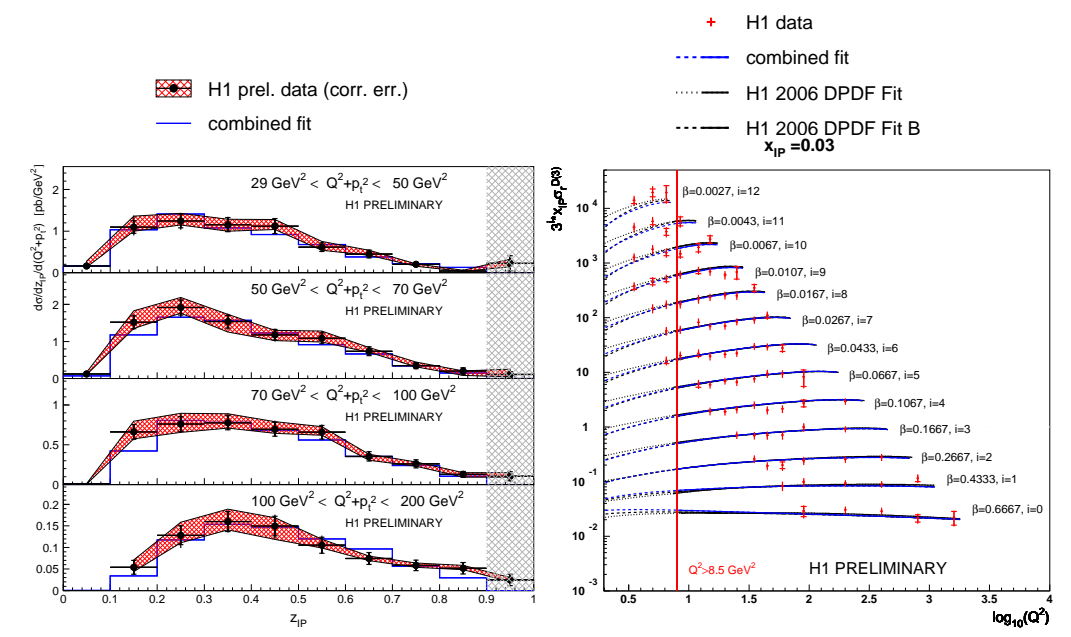

Figure 2. left: Cross section of diffractive dijets doubly differential in in $z_{\mathbb{P}}$ and the scale $\mu=Q^{2}+p_{\perp}^{\text {star } 2}$. Uncertainties are denoted as described in Figure 1 . The blue line shows the NLO QCD prediction based on the combined fit. right: The $\beta$ and $Q^{2}$ dependence of the diffractive reduced cross section $\sigma_{r}^{D(3)}$ multiplied by the pomeron momentum fraction $x_{\mathbb{P}}$ at $x_{\mathbb{P}}=0.03$. The inner and outer error-bars on the data points represent the statistical and total uncertainties, respectively. The data are compared to the results of the combined fit for $E_{p}=820 \mathrm{GeV}$, which is shown as blue lines. The dashed line indicates the prediction in kinematic regions that did not enter into the fit. The two black lines indicate the predictions of the H1 2006 DPDF fit.

The parton densities are parameterized as of momentum fraction $z$ at a starting scale $Q_{0}^{2}$ as $A \cdot z^{B} \cdot(1-z)^{C}$ and evolved to higher scales by the DGLAP equations in NLO. Here, $A, B$ and $C$ are free parameters, determined in the fit. Additionally the Regge intercept $\alpha(0)$ of the pomeron flux factor and the normalization of the sub-leading reggeon exchange enter the fit as free parameters. From these parton densities the reduced cross section for inclusive diffractive DIS is computed in NLO as well as the dijet cross section (using the nlojet++ program). 
The fit has a high quality as shown by the overall value $\chi^{2} / d f=0.89$ which splits into $\chi^{2} / d f=27 / 36$ for the dijet cross sections and $\chi^{2} / d f=$ $169 / 190$ for $F_{2}^{D}$. The resulting parton distributions are shown in Figure 3.

As the NLO QCD DGLAP evolution is able to describe both the shape and scaling violations of $F_{2}^{D}$ and the dijet cross sections consistently, we conclude that QCD factorization in DIS is valid in our kinematic region. The data has allowed for the first time to determine both the diffractive gluon and the singlet quark distribution with good accuracy in the range $0.1<z_{\mathbb{P}}<0.9$.

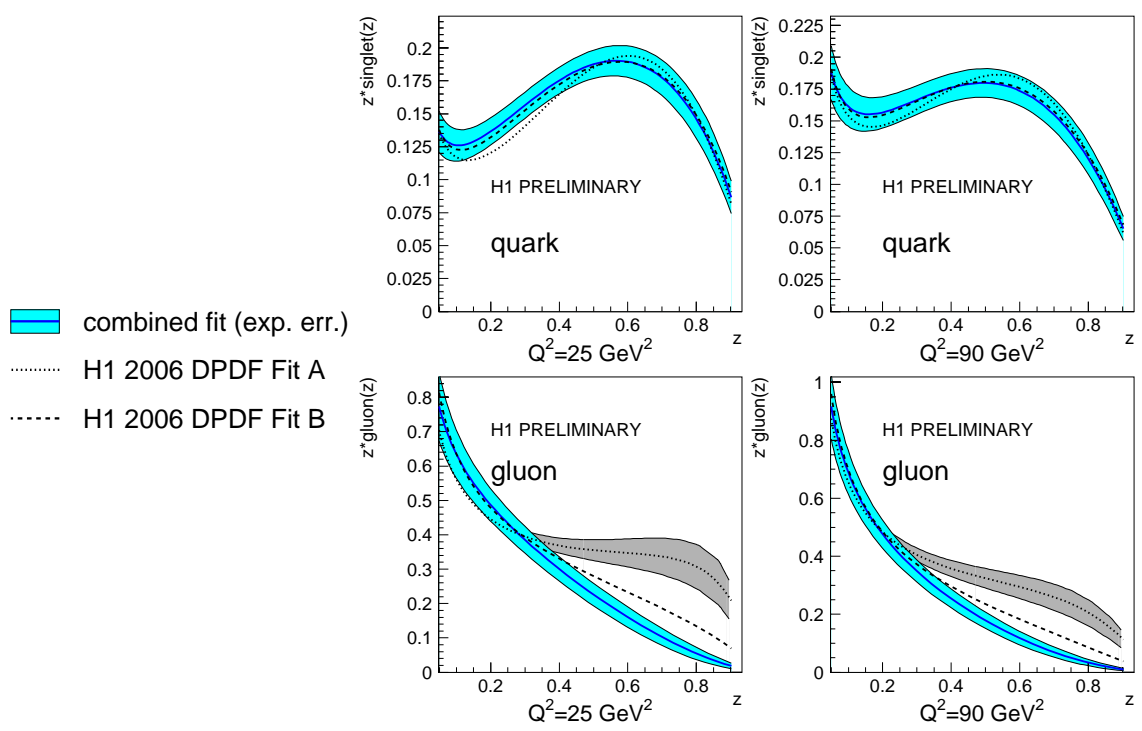

Figure 3. The diffractive singlet density (top) and diffractive gluon density (bottom) for two values of the hard scale $\mu$ : $25 \mathrm{GeV}^{2}$ (left) and $90 \mathrm{GeV}^{2}$ (right). The blue line indicates the combined fit, surrounded by the experimental uncertainty band in light blue. The two dashed lines show the two fit results from [3] for comparison.

\section{References}

1. J. Collins, Phys. Rev. D57 (1998) 3051 and erratum-ibid. D61 (2000) 019902.

2. C. Adloff et al. [H1 Collaboration], Z. Phys. C76 (() 1997) 613

3. [H1 Collaboration], arXiv:hep-ex/0606004.

4. H1prelim-04-113 